\title{
Automobile Engine Compartment Parameters Optimization Based on CFD Simulation
}

\author{
Wei YANG ${ }^{1}$, Shao-Ning WU1 ${ }^{1}$, Wei WANG ${ }^{1}$, Yu YANG ${ }^{2}$, Li LU ${ }^{2, a,{ }^{*}}$ \\ ${ }^{1}$ Shanghai Automotive-General Motor-Wuling Automobile Co., Ltd, TDC,Liuzhou,China. \\ ${ }^{2}$ Wuhan University of Technology, Wuhan,China \\ aluli19920902@163.com \\ ${ }^{*}$ Corresponding author
}

\section{Keywords: Cooling System, CFD, MRF, Engine Compartment.}

\begin{abstract}
This paper illustrates how to use the CFD technique to analyze the cooling system of vehicle engine compartment. For the completeness of the research, the study build a wind tunnel, making the research close to the reality, to simulate two working conditions of vehicle and use the MRF technique in the fan analysis. From the result, the flow field and the temperature field characteristic near the vehicle body and the situation in the engine compartment are confirmed. Furthermore, some valid conclusion are obtained. In order to improve the performance, some improvement schemes, basing on the analysis result, are putted forward. The schemes place guide plates to reform the flow condition in the engine compartment, preventing the vortex appearing to make the flow fluently. Meanwhile, compare the air-inlet rate of the radiator which use different guide plate projects, setting the angle of plate regularly from $0^{\circ}$ to $15^{\circ}$ in $3^{\circ}$ intervals. According to the comparison, these optimize schemes do acquired certain effect.
\end{abstract}

\section{Introduction}

With the development of technology, the requirements of vehicle emissions performance, reliability and fuel consumption, concerning by the whole automobile industry, have become more strictly. The fuel consumption, one of the requests, is directly influence the performance of engine. Meanwhile, the component modular extent dramatically increasing makes the vehicle cabin layout become more compact, which led to an unpleasant heat dissipation condition? Therefore, the research about the cooling system in the vehicle has become the focus of attention. Yang $\mathrm{Z}$ and his team try to find out the differences between two cooling system models (CRFM \&FRM) which layout the condenser, radiator, fan in different order[1]. Gulbarga P constructed an auto cooling system simulation platform to help his systemic research [2]. Using CFD to analyze vehicle flow characteristic now has widely taken by the researcher of automobile industry. Compare to the traditional aerodynamics research method, long cycle and high cost, CFD has many extraordinary advantages. For instance, Computational Fluid Mechanics technique makes the research program more flexible and gets large number of data which the old way can never obtain. It obviously shorter the cycle and lower the fee, by the way. From the result, calculating by the CFD technique, the technicians can obtain a comprehensive understanding about the feature and detail of fluid. Thus, in this paper, the CFD technique is used to research. Concretely, the research employ software Fluent to analysis vehicle flow field, both of the car body and the engine compartment, and provide theoretical basis for further optimization of cooling system, air-inlet grille and the air shaft of engine compartment.

\section{Theoretical Analysis}

The object of this research is a mid-engine design commercial vehicle. Because of the speed of vehicle slower than $400 \mathrm{~km} / \mathrm{h}$, the calculation regards the air as incompressible gas. Meanwhile, the whole process of build flow field control equations must obey various theorem and theory in the engineering thermal physics. 
Momentum conservation equation.

$$
\frac{\partial}{\partial x_{j}}\left(\rho u_{j} u_{i}-\tau_{i j}\right)=-\frac{\partial p}{\partial x_{j}}
$$

Energy conservation equation.

$$
\frac{\partial}{\partial x_{j}}\left(\rho u_{j} h-k \frac{\partial T}{\partial x_{j}}\right)=u_{j} \frac{\partial p}{\partial x_{j}}+\tau_{i, j} \frac{\partial u_{i}}{\partial x_{i, j}}
$$

Compare to the vehicle body, the gas separation, because of the complex construction, is more likely to occur in the engine compartment. Therefore, the air in the engine compartment is the turbulent flow, obeying the turbulent transport equation [3]. Choosing the $k-\varepsilon$ model to when calculate and the velocity and pressure coupling in the discrete equations are solved by SIMPLE method.

Turbulent kinetic energy equation:

$$
\frac{\partial(\rho k)}{\partial t}+\frac{\partial\left(\rho k u_{i}\right)}{\partial x_{i}}=\frac{\partial}{\partial x_{i}} \times\left[\left(\mu+\frac{\mu_{t}}{\sigma_{k}}\right) \frac{\partial k}{\partial x_{j}}\right]+G_{k}+G_{b}-\rho \varepsilon-Y_{M}+S_{k}
$$

Turbulent dissipation rate equation:

$$
\frac{\partial(\rho \varepsilon)}{\partial t}+\frac{\partial\left(\rho \varepsilon u_{i}\right)}{\partial X_{i}}=\frac{\partial}{\partial X_{j}}\left[\left(\mu+\frac{\mu_{t}}{\sigma_{\varepsilon}}\right) \frac{\partial \varepsilon}{\partial X_{j}}\right]+C_{1 \varepsilon} \frac{\varepsilon}{k}\left(G_{k}+C_{3 \varepsilon} G_{b}\right)-C_{2 \varepsilon} \rho \frac{\varepsilon^{2}}{k}+S_{\varepsilon}
$$

\section{Pretreatment}

During the modeling, simplifying the geometry of the vehicle, cleaning up the irrelevant parts, makes the calculation easier, because the research focus on the flow filed of the body and engine compartment. To be specific, importing the geometry, by a ratio of 1 to 1 , into software ANSA where wiping off the needles feature and seaming the separation. After cleaning, display the vehicle model with PID setting, which divide to CAR_SURFACE, and other 14 forms, to lay the foundation of simulation the constructing wind tunnel and setup the boundary condition.

A special space is needed to simulate the flow filed which exactly the same as the condition of vehicle running on the road. Thus, the research builds a wind tunnel. In order to avoid the clogging effect, existing more or less in the wind tunnel simulation, a series of specific size must be followed when building. The length of front space is 3 times of the vehicle, when the back is 5 times. Meanwhile, the space height is 5 times as that of the vehicle, the width of the tunnel taking the equal multiple. The design sketch is displayed in Fig. 1.

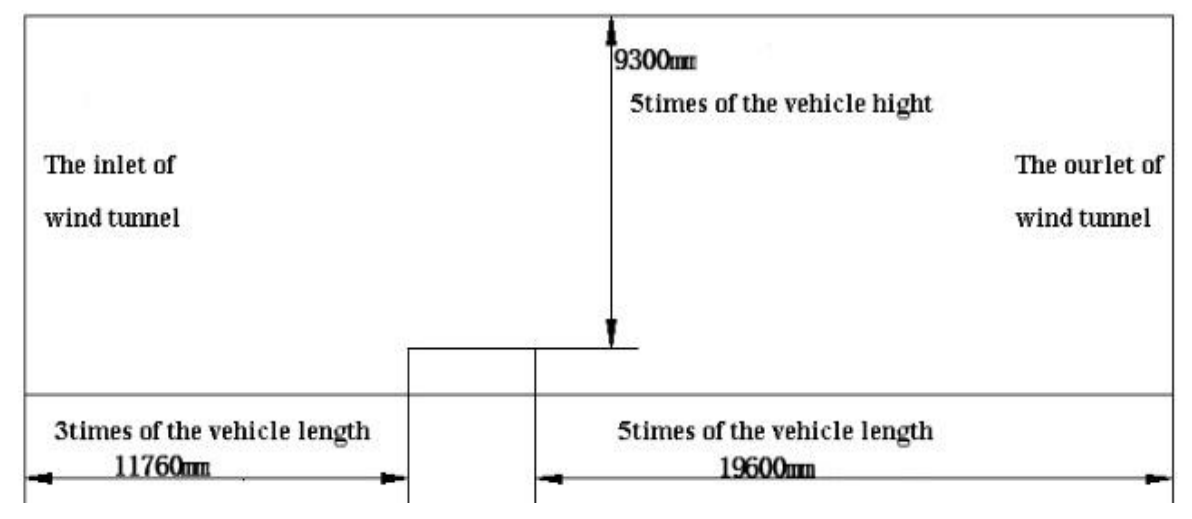

Fig.1. The size of wind tunnel (Front View)

Generating the triangular mesh in ANSA and importing into Fluent to Tet mesh. The condition of liquid in front of the vehicle is lamellar flow, but when it come closer to the air-inlet grille the situation change. In this circumstance, the grid density around the body, when Tet mesh, must be 
increased. The range of increasing density field is $2000 \mathrm{~mm}$ in front of the head, $2800 \mathrm{~mm}$ out to the side, $7300 \mathrm{~mm}$ back of the vehicle. To shortcut the calculation quantity, selecting half of car model for it bilateral symmetry construction. The total number of grid is about 6.58 million, and the max Skewness of grid is 0.9610432 while the Min Skewness of grid is 3.023003e-07and the Avg Skewness of grid is 0.32537215 . The effect is displayed in Fig. 2.

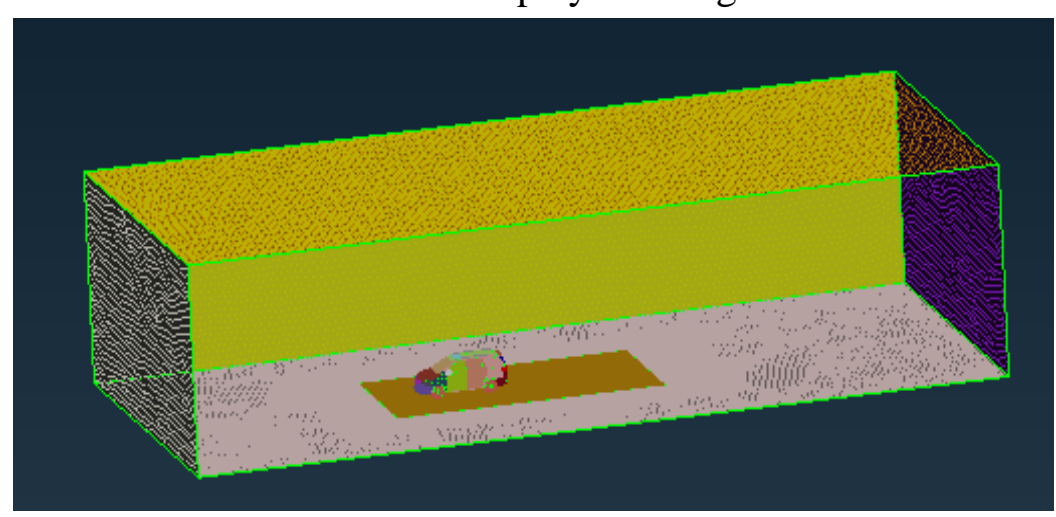

Fig.2. The whole flow field grid

Appropriate parameter setting is necessary which means the boundary condition must fit the reality situation during the simulation.

- $\quad$ The entrance of wind tunnel. Setting the boundary condition into VELOCITY-INLET. The direction of speed and plane is vertical and the environment temperature is $15^{\circ} \mathrm{C}$.

- The exit of wind tunnel. Setting the boundary condition into PRESSURE-OUTLET. Inputting the standard atmospheric pressure into the pressure blank.

- $\quad$ The body and engine compartment of vehicle and the wall of tunnel. Setting the boundary condition, up and grand plane of tunnel, into MOVING WALL, velocity is same as the vehicle, while the unmoving part of body and engine compartment is STATIONARY WALL.

- The radiator and condenser. Setting the boundary condition into POROUS JUMP, meantime adjust the damping factor of component, except normal component, into infinity.

- $\quad$ The fan. The research use MRF analysis technique to calculate the rotary fan field. MRF technique is a simple steady calculating method, calculating by separate the rotating field.

The relationship between the rotating coordinate and the absolute coordinate [4]:

Momentum equation of MRF technique

$$
\rho \frac{d V}{d t}=\frac{\partial}{\partial t}\left(\rho V_{r}\right)+\nabla \cdot\left(\rho V_{\tau} V_{r}\right)+\rho\left(2 \Omega \times V_{r}+\Omega \times \Omega \times r\right)+\rho \frac{\partial \Omega}{\partial t} \times r
$$

Because of the factor $\rho \partial \Omega / \partial t \times r$ always ignored in calculation, the MRF technique can not exactly express the data change by time-dependent. According to the reference, setting the rotating field and adjusting the rotational speed to $2300 \mathrm{rpm}$ while continuous of the velocity vector, the interface of rotation area and steady area, should be guaranteed [5].

Choosing the two typical working conditions of the vehicle, the engine working in the rated power point $(71 \mathrm{~km} . \mathrm{h}-1)$ and the maximum torque point $(45 \mathrm{~km} . \mathrm{h}-1)$, to study the characteristic of cooling system, and research how the temperature field of the whole engine compartment exhibit.

\section{Simulation Analysis}

The analyze result is showed in Fig. 3. The flow produce a shunt at the A area, from the graph, and flow over the body which led to a high pressure higher than the around field. When the flow comes to the B area, the flow become faster and the pressure decrease. That is the reason, the steam coagulation always happened in the B area of vehicle when driving in the low temperature and moist weather. The vortical flows occurred after the flow reaches the $\mathrm{C}$ area where the flow stick to front window and cause the separation bubble. This area relatively stable and has obvious vortex. 
Then, the flow reaches the D area, the top of the vehicle, the liquid moving faster with the pressure drop again. But at the back of $\mathrm{E}$ area, the situation is opposite of $\mathrm{D}$ area, creating an opportunity for the flow separation and the vortex generation. Finally, the flow stick to the back area and bring the separation bubble. Meanwhile, a small vortex field occurred.

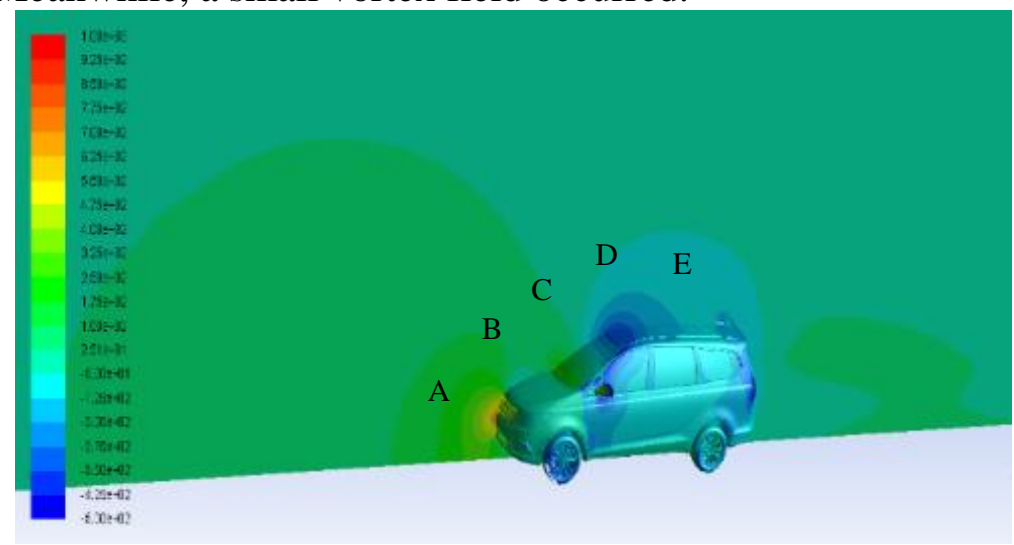

Fig.3. The pressure field of the vehicle body

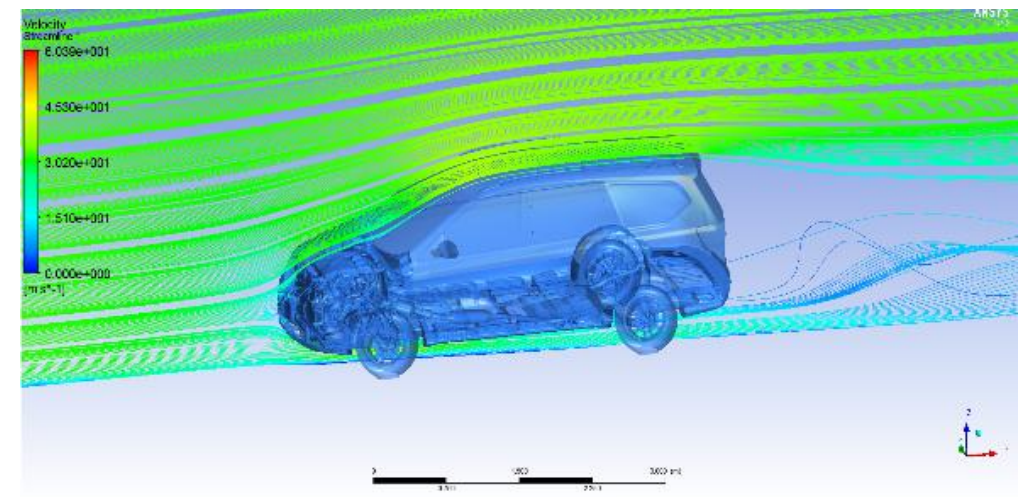

Fig. 4. The flow field streamline of vehicle body

The flow field line is displayed in Fig. 4. The flow pattern have clearly stable flow lines. The laminar flow turned into the main role, and there are a few of turbulence. With common effect of the separation bubble, caused by speed decreasing, and fluid director, there is a vortex field occurred at the upside of back area. Also, there is another vortex field at the bottom, causing by viscous retardant from the ground, which will takes the dirt pollute the back window and resist the vision of driver. The flow near the air-inlet grille presents the laminar flow situation, and a part of that flow, separating from the headstock, enter the engine compartment from the vehicle bottom.

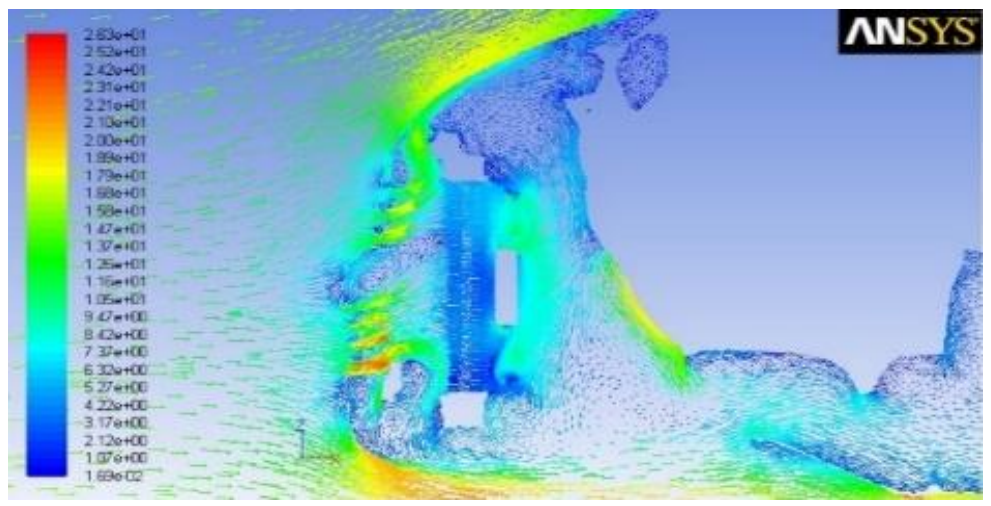

Fig. 5. The velocity vector of engine compartment 


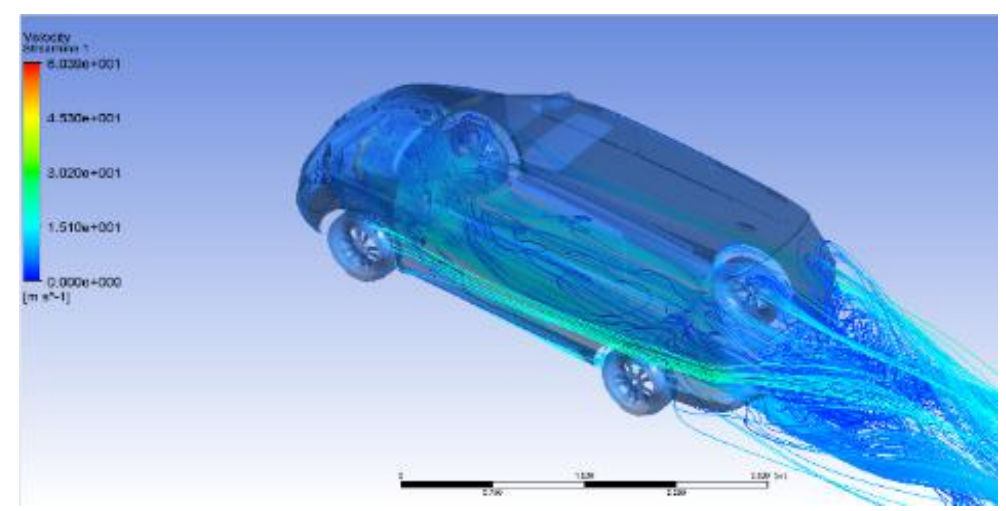

Fig. 6. The flow field streamline

The Fig. 5 shows the situation of flow in working conditions. In these two condition, the flow in the compartment is complex. The flow move into the engine compartment, through the air-inlet grille, cool the condenser by cooperating with the fan. The vortex come into being, between the air-inlet grille and condenser, because the incomplete ventilation of condenser and radiator. Meanwhile, the flow separate both at the upside and the bottom of these two parts. A dramatic increasing speed can be obviously found at the fan boundary. However, the velocity suddenly decreased by the resisting of the nose cabin clapboard and the vortex occurred follow on. Otherwise, there is part of airflow, flowing into the cabin already, separate at the middle of cabin. The flows, which continue flowing to the mid-engine, is become slower, because of the resistance of engine shell. And, the retention phenomenon of gas, happening around the engine, is adverse to the heat dissipation of engine.

The Fig. 6 displays the flow situation without engine compartment shelter, making the observation of complex engine construction and disorder flow condition clearly. From the graph, the whole flow process of liquid shows the fan plays irreplaceable role. The flow state and speed both changed, after passing the fan. When the vehicle at the rated power point, the outside pressure. The value of fan system is negative. Thus, the system needs the radiator draft the cooling flow. However, at the maximum torque point, the fan system limits the flow which flows into the radiator.

\section{Optimization}

The Fig. 7 is airflow diagram of the vertical plane of symmetry, some shortcomings have been circled in the Fig. At the circles 1 and 3, the airflow gets bypassed through the gap between the bumper and the condenser that cannot be used to heat exchange. And, at the circles 2, there is a big swirl between upper and below grille, which will reduce the air-inlet in radiator, all these shortcomings will affect the cooling capacity of the radiator groups.

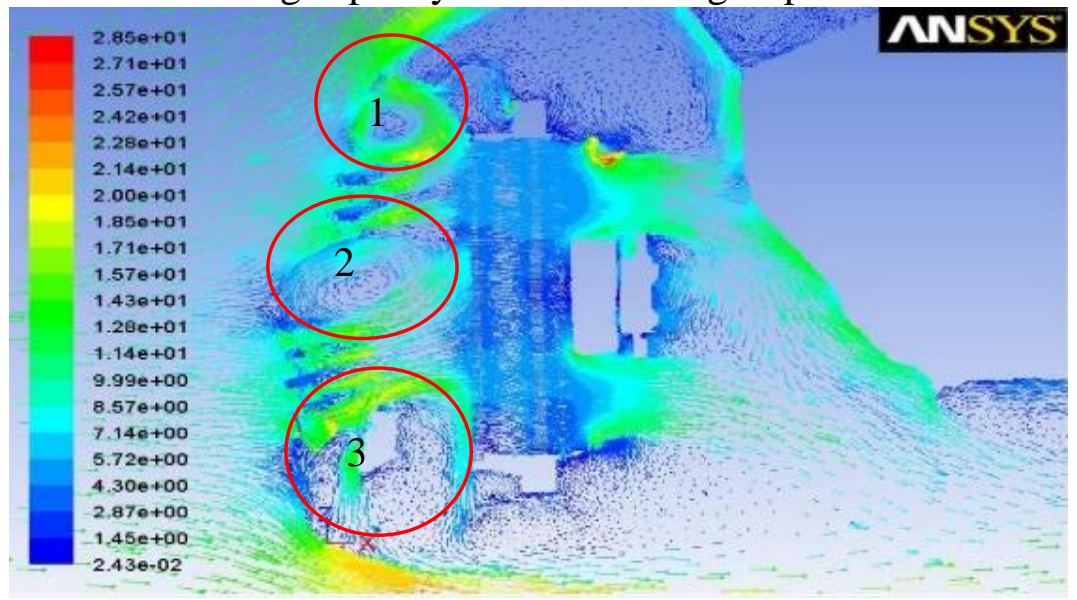

Fig. 7. Airflow diagram of the vertical plane of symmetry 
In order to reduce the airflow getting bypassed through the gap between the bumper and the condenser, the structure called guide plate is installed in the after of the upper and below grille. And, In order to choose the best installation inclination angle of the two guide plates, on the one hand, both guide plates are installation with different angle starting with 0 degree and every scale represents 3 degree to construct 6 simulation model, guide plate are inclined downstream. Then, simulating these ten kinds' models and calculating the rate of air-inlet in radiator .At last, comparing the result with the result of original model. The simulation and comparison result are showed in table 1.Streamline diagram of optimization model is showed in Fig. 8.

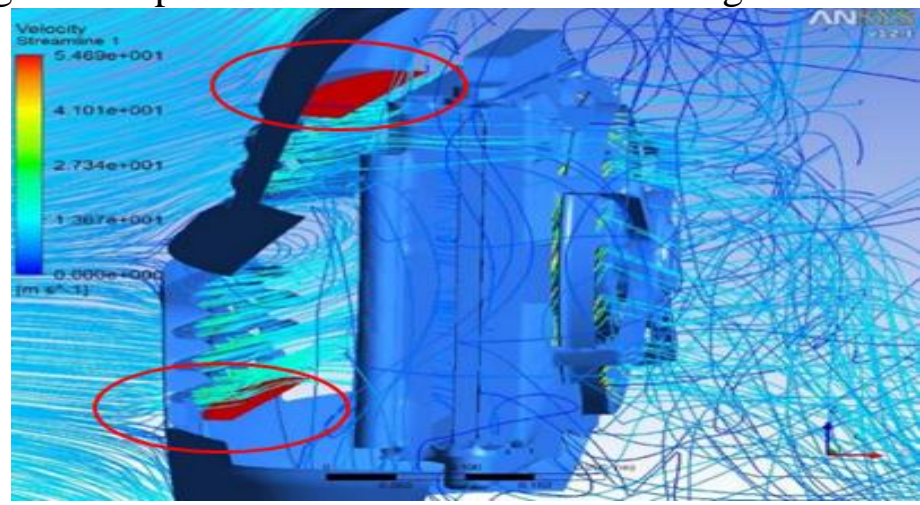

Fig. 8. Streamline diagram of optimization model

By comparing the streamline diagram of original model and optimization model, the conclusion can be easily seen that the guide plate can prevent airflow get bypassed through the gap between the bumper and condenser, as the Fig. 8 shows. In addition, the table 1 shows that when the inclination angle of the both guide plates was 3 degree, the rate of the air-inlet in radiator increased 3.61 percent than original model. All things considered, the guide plate can prevent airflow get bypassed and guide the airflow flow into the radiator groups, different inclination angle will have different result, when the inclination angle of all guide plates are 3 degree, the radiator groups have the best optimization.

Table 1. The simulation and comparison result of the air-inlet (Air-inlet. $\mathrm{kg} . \mathrm{s}^{-1}$ )

\begin{tabular}{|c|c|c|c|}
\hline $\begin{array}{c}\text { Angle of guide } \\
\text { plate }\end{array}$ & $\begin{array}{c}\text { Total } \\
\text { air-inlet }\end{array}$ & radiator & $\begin{array}{c}\text { The increasing rate of } \\
\text { radiator }\end{array}$ \\
\hline original & 1.9759 & 0.9958 & \\
\hline $0^{\circ}$ & 1.9608 & 1.0269 & $3.11 \%$ \\
\hline $3^{\circ}$ & 1.9550 & 1.0318 & $3.61 \%$ \\
\hline $6^{\circ}$ & 1.9399 & 1.0301 & $3.45 \%$ \\
\hline $9^{\circ}$ & 1.9275 & 1.0286 & $3.29 \%$ \\
\hline $12^{\circ}$ & 1.9224 & 1.0280 & $3.23 \%$ \\
\hline $15^{\circ}$ & 1.9064 & 1.0248 & $2.91 \%$ \\
\hline
\end{tabular}

There is a big swirl between upper and below grille, which will reduce the air-inlet in radiator groups. In order to eliminate the swirl between upper and below grille, a new structure called mid guide plate is designed, as show in Fig. 8. There are two kinds of mid guide plate which just have different inclination angle, they are named optimization model 1 and optimization model 2.Then, doing CFD simulation analysis of two kinds optimization model and comparing analysis results with the results of original model. The comparison results are shown in the table 2.By comparing and analyzing the air-inlet of radiator groups and the increasing rate of air-inlet in radiator groups, the optimal structure can be selected. The velocity vector diagram of optimization model is showed in Fig. 9. 


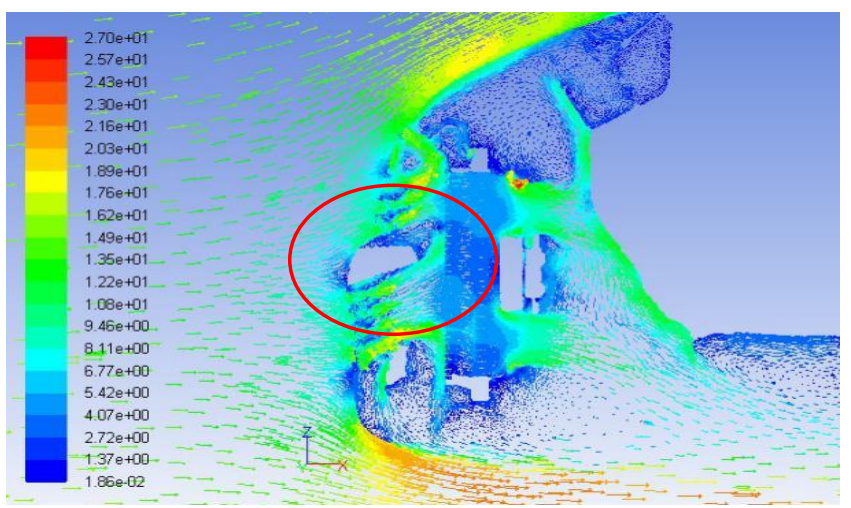

Fig. 9. Velocity vector diagram of optimization model

Table 2. The comparison result of the air-inlet. (Air-inlet. kg.s ${ }^{-1}$ )

\begin{tabular}{|c|c|c|c|}
\hline & $\begin{array}{c}\text { Total } \\
\text { air-inlet }\end{array}$ & radiator & $\begin{array}{c}\text { The increasing rate of } \\
\text { radiator }\end{array}$ \\
\hline Original & 1.9759 & 0.9958 & \\
\hline Optimization 1 & 1.5239 & 0.9920 & $14.70 \%$ \\
\hline Optimization 2 & 2.0600 & 0.9879 & $-2.44 \%$ \\
\hline
\end{tabular}

Table 2 show that the mid guide plate can effectively prevent the swirl formation and the optimization model 1 can effectively increase radiator groups' air-inlet, the increasing rate of air-inlet in radiator is 14.70 percent, so in the two kinds of optimization model, the optimization model 1 is the best.

There is obvious leakage phenomenon in both sides of the radiator, part of the airflow flowing into through the upper grille flow away from both sides of the radiator which cannot be used by radiator groups. In order to prevent airflow flow away, two baffles are installed in both sides of the radiator. Then, doing CFD simulation analysis of the optimization model and comparing analysis result with the result of original model. The comparison results are shown in the table 3 .

Table 3. The comparison result of the air-inlet. (Air-inlet. kg.s-1)

\begin{tabular}{|c|c|c|c|}
\hline & $\begin{array}{c}\text { Total } \\
\text { air-inlet }\end{array}$ & radiator & $\begin{array}{c}\text { The increasing } \\
\text { rate of air-inlet in radiator }\end{array}$ \\
\hline Original & 1.9759 & 0.9958 & \\
\hline Optimization & 1.8442 & 1.0397 & $4.40 \%$ \\
\hline
\end{tabular}

Table 3 shows that the baffles in the both sides of the radiator can effectively prevent the airflow flow away and guide the airflow flow into radiator groups. In a word, the baffles in the both sides of the radiator can increase the rate of air-inlet in radiator, enhance the cooling efficiency.

\section{Conclusion}

Through the CFD simulation analysis of car's front cabin, some shortcomings has been found, in order to optimize these shortcomings, some optimizations for the thermal structure in the car's front cabin have been done. Details and optimization s as follows.

- The airflow gets bypassed through the gap between the bumper and the condenser without going through the heat exchange. By installing upper and below guide plate in the after of the grille and choosing the best angle of the guide plate to guide the airflow, the shortcomings, the airflow getting bypassed, will be solved perfectly, the rate of air-inlet in radiator has effective promotion.

- There is a big swirl between upper and below grille, which will reduce the air-inlet in radiator. By installing mid guide plate in the center of upper and below grille to guide airflow, the swirl can be effectively prevented. The optimization model 1 can effectively increase condenser, radiator and fan's air-inlet and the increasing rate of air-inlet in radiator.

- There is obvious leakage phenomenon in both sides of the radiator, part of the airflow 
flowing into through the upper grille flow away from both sides of the radiator which cannot be used by radiator. By installing baffles in both sides of the radiator to prevent airflow flow away, the leakage phenomenon can be effectively prevented. The rate of the rate of air-inlet in radiator has effective promotion.

\section{Reference}

[1] Z. Yang, J. Bozeman, F.Z. Shen, et al. CFRM Concept at Vehicle Idle Conditions [J]. SAE Paper, (2003)-01-0613.

[2] P. Gullberg, L. Lofdahl, P. Nilsson. Cooling Airflow System Modeling in CFD Using Assumption of Stationary Flow [J]. SAE Paper, (2001)-01-2182.

[3] G.F. Jiang. Research of the Heat Transfer Performance in the Automobile Engine Compartment [J]. Vehicle Technology, 03, (2005).

[4] P. Gullberg, R. Sengupta. Axial Fan Performance Predictions in CFD, Comparison of MRF and Sliding Mesh with Experiments[J]. SAE Paper, (2011)-01-0652.

[5] Y.J. Zhou, H.Y. Xu, W. Kan. Analysis and Numerical Simulation of Flow Field in Centrifugal Pump [J]. Advanced Materials Research, 1006, (2004).

[6] D. Wang, Y. Han. Research and Optimization of Automobile Engine Compartment Heat Dissipation Performance [J]. Automobile technology, 12, (2015), (12).

[7] Y.Y. Feng, Y.L. Li, L.H. Wang. Comprehensive Analysis of Vehicle Thermodynamic and Aerodynamic Performance by Using CFD [J]. Auto engineer, 02, (2015).

[8] X.M. Zhao. Visualization Analysis of the Temperature Field under Engine Hood and Improvement Methods[J].The Chinese mechanical engineering, 14,(2004) .

[9] A. Throat, M. Kulkarni, A. Berg, A. Pisa. CFD Investigation of Airflow through Fan and Cooler System of an Off Road Vehicle [J].SAE Paper, (2013)-01-2792.

[10] G.Q. Liu, W.L. Yang, X.L. Deng. Optimization of under hood Temperature Field for a passage Car [J].The Chinese mechanical engineering, 09, (2013).

[11] P. Cornelius. Evolution of Active Grille Shutters[J].SAE Paper,(2014)-01-0633.

[12] L.P.Li, G.K.Chew, J.M.Dwelling, T.H.Husbandman.C.La. A Flow Approachable to Vehicle Underwood the Heat Transfer Shortcomings [J].SAE Paper, (2015)-01-1073

[13] S. Manna, Y. Kushwah. Optimization of a Vehicle under Hood Airflow Using 3D CFD Analysis [J].SAE Paper, (2015)-01-0349.

[14] Subramaniyan B, Rajaraman R. Numerical Investigation on Airflow Distribution of Automotive Radiator [J]. International Review of Mechanical Engineering, 09, (2015). 